Europhysics Letters

PREPRINT

\title{
Discrete energy landscapes and replica symmetry break- ing at zero temperature
}

\author{
F. $\operatorname{KrzaKala}\left({ }^{*}\right)$ and O.C. $\operatorname{Martin}\left({ }^{* *}\right)$ \\ Laboratoire de Physique Théorique et Modèles Statistiques, \\ Université Paris-Sud, bâtiment 100, F-91405 Orsay, France.
}

PACS. 64.60.Cn - Order-disorder transformations; statistical mechanics of model systems. PACS. 75.10.Nr - Spin glass and other random models.

\begin{abstract}
The order parameter $P(q)$ for disordered systems with degenerate ground-states is reconsidered. We propose that entropy fluctuations lead to a trivial $P(q)$ at zero temperature as in the non-degenerate case, even if there are zero-energy large-scale excitations (complex energy landscape). Such a situation should arise in the 3 -dimensional $\pm J$ Ising spin glass and in MAX-SAT. Also, we argue that if the energy landscape is complex with a finite number of ground-state families, then replica symmetry breaking reappears at positive temperature.
\end{abstract}

Introduction. - A number of disordered and frustrated systems [1] exhibit replica symmetry breaking (RSB) [2]; physically, this means that several macroscopically different valleys contribute to the partition function in the thermodynamic limit. Perhaps the most famous case is the Sherington-Kirkpatrick (SK) model of spin glasses, but RSB is believed to arise in diluted mean field models 3 , f5] too. Furthermore, there is an ongoing debate over the presence of RSB in the Edwards-Anderson (EA) spin glass model in $d \geq 3$. Most Monte Carlo simulations [6] give evidence in favor of RSB there, but it has been objected that the temperatures used $\left(T \approx 0.7 T_{c}\right)$ were too close to $T_{c}$. Also, recent work going to zero temperature [7] suggests that there is no RSB in the $d=3$ case, so this issue remains open.

The order parameter for RSB is the presence of a non-trivial probability distribution for the overlap $q$ between two configurations taken at random according to their Boltzmann weight. In the mean field picture of generic random systems, there are macroscopically different valleys whose free-energies differ by $O(1)$, and thus $P(q)$ is non-trivial. However, since the inter-valley part of $P(q)$ decreases linearly with the temperature $T$ as $T \rightarrow 0$, the zero temperature limit of $P(q)$ is trivial: only intra-valley overlaps survive. It is often believed that this pattern is modified when the energy is a discrete variable and the ground-states are highly degenerate. Indeed, it is common lore that models with discrete energies such as the $\pm J$ EA spin glass or MAX-SAT near but below the transition point [8] should have a non-trivial $P(q)$ if the mean field picture is correct, whereas the presence of a trivial $P(q)$ is considered to validate the droplet model [9]. Here we go against this standard lore, and we will try to convince the

(*) krzakala@ipno.in2p3.fr

$(* *)$ martino@ipno.in2p3.fr

(c) EDP Sciences 
reader that instead of examining $P(q)$ among ground-states it is more appropriate to consider the energy landscape and determine whether there exist macroscopically different valleys. We believe our conclusions apply not only to the 3 -dimensional $\pm J$ EA model, but also to other discrete energy models such as diluted fully-frustrated magnets, MAX-SAT, and diluted mean field $\pm J$ Ising spin glasses to name just a few.

Our first claim is that $P(q)$ in these models is trivial when considering ground-states or even finite energy excited states. Our second claim is that replica symmetry is broken $(P(q)$ is non-trivial) at arbitrarily low temperatures in the thermodynamic limit. In effect, discrete and continuous energy models behave similarly with respect to RSB. At the heart of our arguments is the hypothesis that the effective number of ground-state families does not grow with the system size. (Thus our claims do not apply the 2-dimensional spin glasses.) The presentation that follows is given using the 3-dimensional EA model for definiteness, but the reader can easily see how to rephrase the discussion for any model with a complex energy landscape.

$P(q)$ for ground-states. - In the 3 -dimensional $\pm J$ EA Ising spin glass Hamiltonian,

$$
H=-\sum_{<i j>} J_{i j} S_{i} S_{j}
$$

the $J_{i j}$ are quenched random variables, $J_{i j}= \pm 1$, that couple nearest neighbor spins on a lattice of size $L \times L \times L$. The $J_{i j}$ are discrete so the energy is also discrete. Furthermore, the local field felt by any spin can have the values $\pm 6, \pm 4, \pm 2,0$ only. A field value of 0 leads to a spin that can flip without changing the energy. Since a finite fraction of the spins on the lattice have this property, there is a finite entropy per spin at the ground-state energy level: the number of different ground-states grows at least exponentially with the lattice volume.

A question of central importance is whether these ground-states differ macroscopically. Unfortunately, this question is ambiguous because of the finite density of zero-energy droplets: if we flip all the spins in zero field, do we reach a macroscopically different ground-state? We would like the answer to be no. To make things simple, suppose for this discussion that $L=\infty$; if all the ground-states differ only by zero-energy droplets containing say $1,2, \ldots$, $k$ spins, then we can say that they form a single family of ground-states. In that case, one expects $P(q)$ to be a delta function at $q_{E A}<1$. (Here and in all that follows, our statements are made modulo the up-down symmetry which flips all the spins.) If we let $k \rightarrow \infty$, we still have a single family of ground-states provided the frequency of zero-energy droplets decreases sufficiently rapidly as their size $k$ grows. This is what happens in the "hidden ferromagnet" picture of spin glasses due to Fisher and Huse [9]. In the same vein, when the ground-states form multiple families, one can hope that the ground-states belonging to the same family will differ by finite size droplets only whereas ground-states from different families should differ by at least one infinite zero-energy droplet, plus any number of finite size zero-energy droplets.

When $L$ is finite, it is even more difficult to define ground-state families satisfactorily because zero-energy droplets can arise with as large a $k$ as one wants; there is thus inevitably some additional arbitrariness in one's definition of these families. One rather simple definition was proposed and used by Hartmann [7]: two ground-states are in the same family if it is possible to go from one to the other by a sequence of zero-energy droplets of size $k=1$. But this also means that two ground-states differing by a zero-energy droplet of size 2 may be put into two different families. Thus it seems more appropriate to define families by clustering together ground-states when their overlap is "sufficiently large". In the case of one-step RSB, this is quite clean, the overlaps being either small or large. When confronted with continuous RSB though, the overlaps cannot naturally be split into small and large values: there are families 

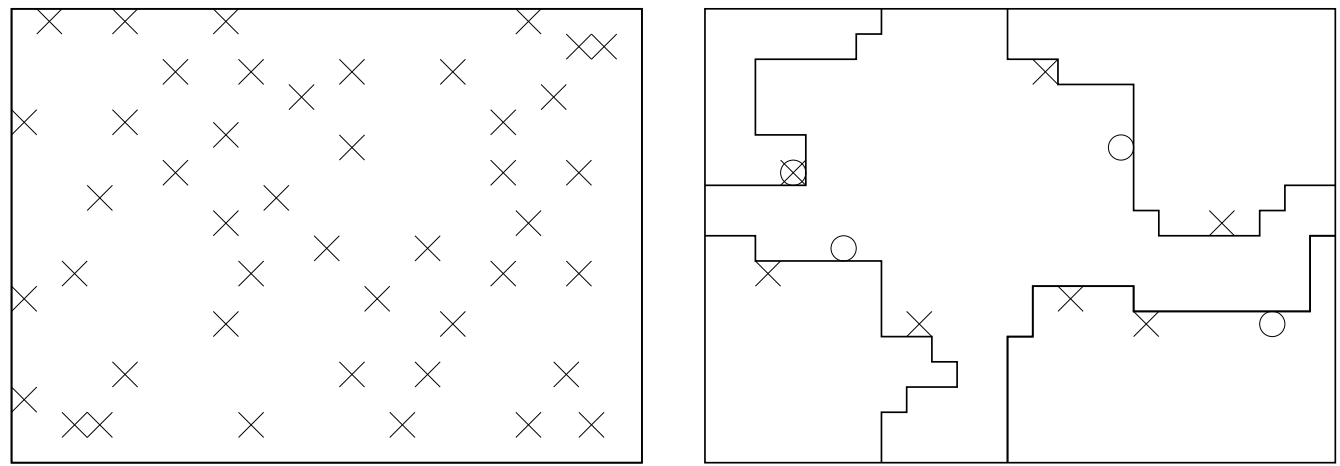

Fig. 1 - a: All spins of ground-state 1 having zero local field (crosses). b: We flip a system-size connected cluster and reach ground-state 2 ; the solid line is the boundary of that cluster. Only those zero local field spins that touch the boundary are relevant for the difference in entropies of the two families of ground-states.

within families ad infinitum, and a cut-off on the overlap has to be introduced, otherwise the number of families will necessarily be infinite. Because of this arbitrariness, the clustering is less elegant than for one-step RSB; nevertheless, this clustering-based procedure can and has been implemented by Hed et al. [10]. Their conclusion, as well as Hartmann's [11], who sampled $P(q)$ amongst ground-states, is that there exists multiple macroscopically distinct families with a finite (non-zero) probability as $L \rightarrow \infty$.

Given such a clustering-based definition of ground-state families, we shall assume hereafter that the model under consideration can have multiple families and thus there is a finite probability of having macroscopically different ground-states. If, when taking the large $L$ limit, this definition of families is good, one may for convenience qualitatively think of a family as all those ground-states obtained from a given one by flipping zero-energy droplets of finite size. On the contrary, when going from one ground-state family to another, it is necessary to flip a "system-size" connected cluster of spins that contains a finite fraction of the whole system. This is illustrated schematically in Figure 1.b where the solid lines represent the boundary $B$ of such a system-size cluster. (The picture is for a cross-section of the 3-dimensional lattice.)

In this kind of system, what does the distribution $P(q)$ of ground-state overlaps look like? For simplicity, suppose that there are just two families, containing $\mathcal{V}_{1}$ and $\mathcal{V}_{2}$ ground-states. For the purpose of comparing the $\mathcal{V}_{i}$, we consider that each family is obtained from one reference ground-state $\mathcal{C}_{i}$ by flipping any number of its zero-energy droplets, taken to be nonadjacent (and thus non-interacting). Let $\mathcal{N}_{i}^{(k)}$ be the number of zero-energy droplets of size $k$ of $\mathcal{C}_{i}$. Then we have $\log _{2} \mathcal{V}_{i}=\mathcal{N}_{i}^{(1)}+\mathcal{N}_{i}^{(2)}+\cdots ;$ clearly $\mathcal{V}_{i}$, which is crucial for the structure of $P(q)$, is very sensitive to the exact number of zero energy droplets in the family. We expect $\mathcal{N}_{i}^{(k)}$ to be self-averaging, growing linearly with $L^{3}$. But there are also fluctuations in this number; if the central limit theorem holds, they will scale as $L^{3 / 2}$, so that the total number of zero-energy droplets $\mathcal{N}_{i}$ of $\mathcal{C}_{i}$ has the form

$$
\mathcal{N}_{i}=\sum_{k} \mathcal{N}_{i}^{(k)}=\alpha L^{3}+x_{i} \sqrt{f L^{3}}
$$

where $x_{i}$ is a (Gaussian) random variable. $P(q)$ has both inter and intra-family contributions. There are $\mathcal{V}_{1}^{2}+\mathcal{V}_{2}^{2}$ ground-state pairs giving the intra-family part, and $2 \mathcal{V}_{1} \mathcal{V}_{2}$ pairs giving the 
inter-family part. We then see that the intra-family part dominates the inter-family part by a factor $2^{\left(x_{1}-x_{2}\right) \sqrt{f L^{3}}}$ (we took $x_{1}>x_{2}$ ). Since this factor diverges as $L \rightarrow \infty$, we find no RSB.

Upon closer examination, we see that it is necessary to be more careful because maybe $x_{1} \approx x_{2}$. In Figure 11.a we show by crosses the spins of ground-state $1\left(\mathcal{C}_{1}\right)$ having zero local field whose number is $\mathcal{N}_{1}^{(1)}$. Figure 1. b shows the boundary $B$ of the system-size cluster that takes one from family 1 to family 2 . It is evident that except for the sites touching $B$, the spins having zero local field are the same in family 1 and in family 2 . These spins then do not contribute to the difference $\mathcal{N}_{1}^{(1)}-\mathcal{N}_{2}^{(1)}$. However, for the spins that do touch $B$, their local field can and usually will be different in the two families. Denoting by $\mathcal{A}$ the area of $B$ (in fact the number of spins touched by $B$ ), the argument that lead to Eq. 2 suggests that $\mathcal{N}_{1}^{(1)}-\mathcal{N}_{2}^{(1)}$ scales instead as $\sqrt{\mathcal{A}}$. Extending this to all droplet sizes, we obtain

$$
\mathcal{N}_{1}-\mathcal{N}_{2} \approx y_{12} \sqrt{\mathcal{A}}
$$

where $y_{12}$ is another (Gaussian) random variable with finite variance. We then see that the previous claim still holds, namely that intra-family contributions dominate the inter-family ones because $\mathcal{A}$ diverges when $L \rightarrow \infty$. (By hypothesis, the number of spins that are flipped when going from one family to another scales as $L^{3}$, so $\mathcal{A}$ grows at least as $L^{2}$.)

This presentation was given for two ground-state families. Extending it to any finite number of families is straight-forward; what really matters for the argument to go through is for $\mathcal{N}_{i}-\mathcal{N}_{j}$ to diverge for any pair of families. This will happen as long as the number of families does not grow too quickly with $L$. To stay as simple as possible, we will continue to assume that the model has a finite number of ground-state families. Then the infinite volume limit of $P(q)$ is trivial (delta functions at $\pm q_{E A}$ ) because of entropy fluctuations amongst the different families. But the trivial nature of $P(q)$ should not be considered as evidence against having finite-energy large-scale excitations $(\theta=0$ in the language of the droplet model), nor against the mean field picture for that matter, as we shall soon see. Finally, there is every reason to expect the reasoning to apply to all discrete models, on lattices or not. For instance in the $\pm J$ Viana-Bray model, $\mathcal{A}$ scales as the total number of spins, so the triviality of $P(q)$ for ground-states should be easier to see than in the EA case.

In a numerical study, the factor $2^{y_{12} \sqrt{\mathcal{A}}}$ should be large. Then for most settings of the disorder variables $J_{i j}$, the inter-family peak in the distribution $P_{J}(q)$ of ground-state overlaps will be small compared to the intra-family term. However, $y_{12}$ has a finite probability density at 0 , so with probability $1 / \sqrt{\mathcal{A}}$ the two peaks will be of comparable size. Thus the $L$ dependence of $P(0)\left(P_{J}(0)\right.$ averaged over disorder) should go as $1 / \sqrt{\mathcal{A}}$ which is faster than $1 / L$. Hartmann [7] indeed finds that $P(0)$ decays, and his fits indicate a $1 / L^{1.25}$ dependence. Taken at face value within our framework, this means that $\mathcal{A}(L) \approx L^{2.5}$.

In the picture we have reached, $P(q)$ is trivial but the energy landscape is "complex"; by that we mean that macroscopically different ground-states appear (taking into account of course the global up-down symmetry). The numerical results [0, 10,11] in support of this picture no longer seem mysterious from a mean field perspective, and lead us to conclude that models with discrete and continuous energies behave similarly: neither have RSB at $T=0$. Note that RSB at $T=0$ implies a complex energy landscape, but the reverse is not true.

$P(q)$ for excited states. - The arguments we gave go beyond just ground-states. Consider the energy landscape of the system, and think of it as composed of different valleys. In this section, we focus on those valleys whose bottom energies are less or equal to some arbitrary fixed cut-off $E_{\max }$. We can consider $P(q)$ for overlaps either among the bottoms of these valleys, or among all spin configurations of energy $E \leq E_{\max }$. (Note that the configurations 
are not weighted by a Boltzmann factor.) Interestingly, the result is the same in both cases, so let us begin with the first one which is simpler.

When $E_{\max }$ is equal to the ground-state energy, the valley bottoms considered are precisely the set of ground-states, and we have clustered these into families. Since defining these families required some arbitrary choices, we see that the same must be true for defining valleys. Thus let valleys be also defined by clustering according to overlaps. The we generalize to valleys a property we used before for ground-state families: for each discrete energy level $E_{i} \leq E_{\max }$, we assume that there is a finite probability of having multiple valleys whose bottom energy is $E_{i}$. Furthermore, as before, we suppose that the number of such valleys remains finite as $L \rightarrow \infty$. Then, regardless of their energy, the valley bottoms play the same role as the ground-state families of the previous section. In consequence, when comparing any two valley bottoms, one will dominate the other. The situation is thus identical to the one we had when looking only at ground-states, and we conclude that $P(q)$ for overlaps between the valley bottoms is trivial as $L \rightarrow \infty$.

Two comments are in order. First, which valley dominates is a random process. As these valleys have energies differing only by $O(1)$, they should have the same statistical properties, so the probability of dominating should be uniform among the different valleys. But since we also expect the typical number of valleys to increase with energy, the winning valley will most likely be near the cut-off $E_{\max }$. The second comment is that since entropy fluctuations lead to single valley dominance, the reasoning suggests that $P(q)$ is trivial at positive temperatures also. This extrapolation will turn out to be too naïve: having a positive temperature requires taking $E_{\max }$ to $\infty$, and then we have to deal with an infinite number of valleys. Before looking into that case, let us go on and investigate as promised the effet of positive energy excitations within the valleys.

Thus, we now consider all the configurations satisfying $E \leq E_{\max }$. We can estimate the size of the valleys (bottoms and higher energy configurations) by including the possibility of flipping positive energy droplets. Because the energy is discrete and bounded by $E_{\max }$, for each valley we can only flip a finite number of such droplets. If we consider all the configurations obtained from a valley bottom by flipping $k$ such droplets, we find that their number is $O\left(L^{3 k}\right)$ times larger than the number at the bottom of the valley. Obviously this power-law factor cannot beat the $2^{y_{i j} \sqrt{\mathcal{A}}}$ factor, and positive energy droplets lead to "negligible" corrections to the valley entropies. The fundamental reason is that the cut-off $E_{\text {max }}$ puts a bound on the number $k$ of positive energy droplets that can be flipped.

$P(q)$ at a positive temperature. - When $T>0$, the number of positive energy droplets that can be simultaneously excited grows linearly with the volume of the lattice. Since we have no reason to excite a finite size droplet of energy $E$ rather than a system-size cluster of the same energy, we see that we have to let $E_{\max }$ go to $\infty$. Then we have a infinite number of valleys to consider! To make progress, we must describe how these valleys are distributed and then estimate their contributions to the partition function.

As was done implicitly when discussing the excited states in valleys, we heuristically view a valley as a ground-state plus all of its possible (finite size) droplet excitations. This is certainly only part of the picture even when just considering the ground-state energy level, but it will do for our presentation. We begin with a statistical description of the number of valleys whose bottoms are at the energy level $E$. In our complex energy landscape, there are more and more such valley bottoms as $E$ grows; we denote by $\rho_{E}(E)$ their density. In the "random energy model" [12] and in the SK model, this density grows exponentially with $E$; 
EUROPHYSICS LETTERS

here we need not be so explicit, we just take

$$
\rho_{E}(E)=e^{S(E)}=e^{S\left(E^{*}\right)+\left(E-E^{*}\right) S^{\prime}\left(E^{*}\right)+\cdots}
$$

where $S(E)$ is a smoothly growing function and $E^{*}$ is any large argument. The important hypothesis we make is that $S(E)$ does not grow with $L$; this must be the case if there is to be a finite number of valleys whose bottoms are at the different (discrete) values of $E$.

Now we want to determine the density $\rho_{F}$ of free-energies of these valleys when the temperature is $T$. First, the free-energy $F_{i}(T)$ of valley $i$ is defined via:

$$
e^{-\frac{F_{i}(T)}{T}}=e^{-\frac{E_{i}}{T}} \sum_{\mathcal{C}} e^{-\frac{E(C)-E_{i}}{T}}
$$

where $\sum_{\mathcal{C}}^{\prime}$ denotes the sum over all configurations $\mathcal{C}$ belonging to valley $i$, and $E_{i}$ is the energy of the valley's bottom. Second, we make the hypothesis that the free-energy $F_{i}(T)$ of valley $i$, when measured with respect to the energy $E_{i}$ at its bottom, is a random variable behaving for instance according to what the central limit theorem would predict were it applicable:

$$
F_{i}(T)=E_{i}+L^{3} f(T)+x_{i} \sqrt{L^{3}}
$$

Here $f(T)$ is the (self-averaging) free-energy density, and $x_{i}$ is a (Gaussian) random variable whose variance depends on $T$. This ansatz is based on the idea that the valleys are statistically similar, and so the second factor on the right-hand-side of Eq. 5 has no statistical dependence on the value of $E_{i}$. Put simplistically, there is no way to know within one of these valleys whether or not it contains the ground-state.

Given our ansatz, the density $\rho_{F}(F)$ of the valley free-energies can now be computed. From Eq. 6, $\rho_{F}(F)$ is obtained by a convolution of $\rho_{E}(E)$ with a Gaussian distribution $G(x)$ :

$$
\rho_{F}(F)=\int_{-\infty}^{\infty} \rho_{E}(E) d E \int_{-\infty}^{\infty} G(x) \delta\left(F-E-L^{3} f(T)-x \sqrt{L^{3}}\right) d x
$$

First, use the $\delta$ function to perform the integral over $x$. Then, because $\rho_{E}(E)$ grows steeply, the integral's main contribution comes from the neighborhood of $E^{*}$, a large value of $E$. Finally, we use Eq. 田; defining $1 / T^{*}=S^{\prime}\left(E^{*}\right)$, the integrand becomes a Gaussian in E. Performing the integral then leads to

$$
\rho(F)=e^{\frac{F-F^{*}(T)}{T^{*}}}
$$

where $F^{*}(T) \approx L^{3} f(T)$ is a reference free-energy. The important point is that $\rho_{F}(F)$ grows exponentially with $F$ : we obtain exactly the form given by the random energy model and by mean field theory. For such a $\rho_{F}$, the lowest (valley) free-energies differ by $O(1)$, and so multiple valleys contribute to the partition function even as $L \rightarrow \infty$. The conclusion is that for positive temperatures below $T^{*}$, there is RSB, and $P(q)$ is non-trivial.

After all this is said and done, we realize that we have made the same mistake here as when we estimated $P(q)$ for ground-states. Indeed, in Eq. 6, the $x_{i}$ of the different valleys are correlated. What is relevant is the scaling of the differences in the valley free-energies. Generalizing the argument used for ground-states, we replace Eq. 6 by

$$
F_{i}(T)-F_{j}(T)=E_{i}-E_{j}+y_{i j} \sqrt{\mathcal{A}_{i j}}
$$

Taking any scaling form for $\mathcal{A}_{i j}$, we substitute in Eq. 7 the term $x \sqrt{L^{3}}$ by a term $y \sqrt{\mathcal{A}(L)}$; up to unimportant prefactors, this leads to the same exponential law for $\rho_{F}$ as before, and thus to RSB. 
It is also possible to extend the calculation using a very different ansatz. Assume that energy differences between valley bottoms are not $O(1)$ but instead scale as $L^{\theta}$. Then one finds that the free-energy differences also scale as $L^{\theta}$.

Summary and conclusions. - In a general system with quenched disorder, we say that its energy landscape is "complex" if there are macroscopically different valleys with $O(1)$ energy differences. When the energy is discrete, (as in the $\pm J$ Ising spin glasses), the ground-states can be highly degenerate, but we expect to be able to clusterize them into families. If the number of such familiess remains finite in the infinite volume limit, then we expect just one family to dominate all the others by its size. This effect is simply due to entropy fluctuations among the different families, and leads to the absence of replica symmetry breaking at zero temperature. The absence of RSB (that is a trivial overlap distribution $P(q)$ ) at zero temperature does not necessarily validate the droplet model. Instead, our over-all message can be summarized as follows: "A system having valleys with $O(1)$ energy differences (a complex energy landscape) should lead to a free-energy landscape with $O(1)$ free-energy differences, and thus to RSB". It is thus not relevant to look at $P(q)$ at zero temperature, nor even $P(q)$ among finite energy excited states; the energy landscape seems to be a better object to consider when trying to distinguish the droplet and mean-field pictures. Amuzingly, we see that the discrete nature of the energies plays no role in determining whether there is RSB or not, be-it at zero or at finite temperature.

Our point of view allows one to understand a number of numerical results on the $\pm J$ EA model [7, 11]. Furthermore, it suggests that when going to very low temperatures [13], it is important to control the limit $L \rightarrow \infty$ before taking $T \rightarrow 0$. Finally, we predict that a careful study of ground-states in MAX-SAT will give a trivial $P(q)$ rather than a one-step RSB pattern.

$* * *$

We thank H. Hilhorst and E. Marinari for discussions and J.-P. Bouchaud for his detailed comments. F.K acknowledges financial support from the MENRT, and O.C.M. support from the Institut Universitaire de France. The LPTMS is an Unite de Recherche Mixte de l'Université Paris XI associée au CNRS.

\section{REFERENCES}

[1] Spin Glasses and Random Fields, edited by A. P. Young (World Scientific, Singapore, 1998).

[2] M. Mézard, G. Parisi, and M. A. Virasoro, Spin-Glass Theory and Beyond, Vol. 9 of Lecture Notes in Physics (World Scientific, Singapore, 1987).

[3] L. Viana and A. J. Bray, J. Phys. C 18, 3037 (1985).

[4] J. R. Banavar, D. Sherrington, and N. Sourlas, J. Phys. A Lett. 20, L1 (1987).

[5] M. Mézard and G. Parisi, Europhys. Lett. 3, 1067 (1987).

[6] E. Marinari et al., J. Stat. Phys. 98, 973 (2000), cond-mat/9906076.

[7] A. Hartmann, Euro. Phys. Jour. B 13, 539 (2000).

[8] G. Biroli, R. Monasson, and M. Weigt, Euro. Phys. J. B 14, 551 (2000).

[9] D. S. Fisher and D. A. Huse, Phys. Rev. B 38, 386 (1988).

[10] G. Hed, A. K. Hartmann, D. Stauffer, and E. Domany, cond-mat/0007356.

[11] A. Hartmann, Europhys. Lett. 40, 429 (1997), cond-mat/9704187/.

[12] B. Derrida, Phys. Rev. B 24, 2613 (1981).

[13] H. G. Katzgraber, M. Palassini, and A. P. Young, cond-mat/0007113. 